\title{
Urbanización de favelas: ¿viejas nuevas precariedades?
}

\author{
Maria de Lourdes Zuquim \\ Universidad de São Paulo, Facultad de Arquitectura y Urbanismo, \\ Napplac, Brasil \\ Ana Júlia Domingues das Neves Brandão \\ Universidad Nove de Julho, Brasil \\ Universidad de São Paulo, Facultad de Arquitectura y Urbanismo, \\ Napplac, Brasil \\ Recibido: 30 de junio del 2017 / Aprobado: 6 de septiembre del 2017 \\ doi: 10.26439/limaq2018.n004.2260
}

Uno de los grandes problemas de las ciudades brasileñas es la precariedad —urbana, social y ambiental — en la que vive gran parte de su población. La urbanización del país se consolidó por medio de un proceso excluyente y desigual, que niega a un sector de la sociedad el acceso a la tierra, la vivienda y la ciudad. El enfrentamiento de esta cuestión adquirió una dimensión inédita en la política urbana y habitacional a partir del 2000. Los avances legales y programáticos fueron muchos, así como las intervenciones en el espacio urbano precario. En este artículo se discute la urbanización de dos favelas en el municipio de São Paulo:Vila Nova Jaguaré y Sapé. Además, se investigan las transformaciones urbanas introducidas en el programa a la luz de dos preguntas centrales: ¿la favela se convirtió en ciudad? ¿Cuáles son los nuevos rostros de la precariedad?

precariedad urbana, urbanización de favelas, habitación, proyecto urbano, São Paulo

\section{Urban development of favelas: old new precariousness?}

One of the great problems of Brazilian cities is the precariousness - urban, social and environmental - in which a large part of their population lives. The country's urban development was consolidated through an exclusive and unequal process, which denies a sector of the society access to land, housing and the city. On the other hand, confronting this issue has acquired an unprecedented dimension in urban and housing policy since the 2000s. Legal and programmatic advances were many, as well as interventions in the precarious urban space. This article discusses the urban development of two favelas in the municipality of São Paulo, Vila Nova Jaguaré and Sapé, and looks into the urban transformations introduced in the program in the light of two central questions: Did the favela become a city? What are the new faces of precariousness?

urban precariousness, urban development of favelas, lodging, urban project, São Paulo 


\section{INTRODUCCIÓN}

Pensar en ciudades brasileñas es pensar en favelas, partes inseparables del proceso de urbanización brasileño, que es incompleto, desigual y excluyente.

Históricamente, la entrega de vivienda social estuvo muy por debajo de la demanda de la población de las clases populares; y esta, sin acceso a una casa y a la tierra urbanizada, no tuvo más opción que recurrir a soluciones propias: tugurios, alquiler, parcelaciones irregulares y favelas ${ }^{1}$.

En ausencia de producción estatal y provisión formal (mercado) suficientes para atender a las clases populares, las parcelaciones periféricas y las favelas han pasado a ser las formas predominantes en que los pobres viven en las ciudades $^{2}$, y retratan el proceso de urbanización incompleto y precario común en las ciudades brasileñas.

En São Paulo este proceso se dio en dos tiempos distintos. El primero entre 1940 y 1970, con las parcelaciones populares periféricas producidas sin infraestructura, sin instalaciones sociales y distantes de los servicios públicos y de los puestos de trabajo. Este proceso fue el motor de la urbanización periférica ${ }^{3}$.

El segundo, con la intensificación del surgimiento de favelas a partir de la década de 1970, años de sucesivas reestructuraciones económicas, ajustes salariales, desempleo, aumento en el costo de vida y valorización del precio de la tierra, en los que el lote popular se volvió prácticamente inasequible para los sectores de bajos ingresos. Sin otra alternativa, la solución fue ocupar zonas de uso común en las parcelaciones populares, habitar nuevas áreas centrales o periféricas y, además, tugurizar favelas antiguas. Como ejemplo,

1 Ver Bonduki (1998).

2 Las favelas surgieron en diferentes épocas por todo el Brasil: en São Paulo, en la década de 1940; en Salvador, con invasiones colectivas de la tierra; en Río de Janeiro, aun a finales de la década de 1900 con la remodelación urbanística de la ciudad; en Belo Horizonte, en la segunda mitad de la década de 1890, con la construcción de la ciudad; y así sucesivamente en los demás grandes centros urbanos (Pasternak, 1997).

3 Ver Mautner (1990). 
en 1973 las favelas representaron apenas el $1 \%$ de la población del municipio, mientras que en 1987 pasaron a ser el 8,92\% (Pasternak, 2006).

Si bien en un primer momento las parcelaciones populares periféricas siguieron el tradicional crecimiento centro-periferia; después, las favelas continuaron con la intensificación de la ocupación del suelo urbano. Aunque ambas presenten procesos urbanos y formas de la precariedad comunes, son distintas las orientaciones de la intervención pública ${ }^{4}$ en estos espacios. Mientras que las directrices para las parcelaciones son principalmente de regularización rural o urbanística, para las favelas van más allá de estas, con la ejecución de importantes obras de urbanización.

Los siguientes datos ilustran el panorama actual de las favelas en São Paulo: hoy más de 1300000 personas viven en favelas y 1700000 en parcelaciones irregulares; respectivamente, el 11,6 \% y 15,2 \% de la población paulista (Centro de Estudos da Metrópole, 2016). La mayoría de las favelas se encuentran en las regiones sur $(53,2 \%)$ y este $(26,17 \%)$ del municipio de São Paulo (figura 1). De forma frecuente están en áreas ambientalmente frágiles del territorio paulista sea en laderas o en el fondo de los valles con un patrón de ocupación compuesto de lotes, manzanas y vías irregulares, lo que dificulta el acceso y el tráfico de vehículos y personas (IBGE, 2010). La vulnerabilidad social y la susceptibilidad de los terrenos, sumadas al patrón constructivo precario y fuera de las normas edilicias, han llevado a deslizamientos en el caso de las laderas; o de erosiones, especialmente en las márgenes de ríos y riachuelos.

Estas características, que combinan fragilidad ambiental e irregularidades físico-urbanísticas y jurídicas, determinan las formas de intervención que estas áreas podrán sufrir como consecuencia de las políticas públicas.

4 Aunque tanto las favelas como las parcelaciones presenten irregularidad de ocupación del suelo urbano, irregularidad rural y la autoconstrucción como forma de provisión de la vivienda y autoprovisión de infraestructura, es el acceso a la tierra lo que las distingue. Mientras que la Iotización irregular se dio con algún tipo de parcelación del suelo y de transacción de compra de la tierra; la favela, por lo general, surge por la ocupación progresiva y organizada de áreas de dominio público o privado, sin acciones de parcelación del suelo. 


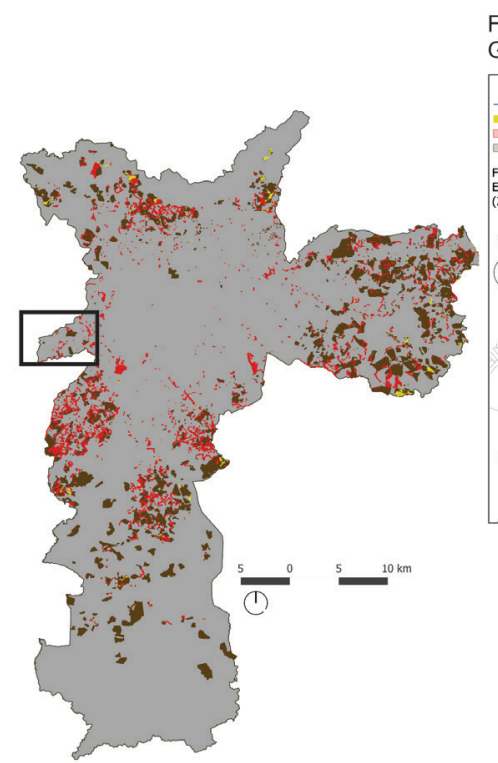

Favelas, Loteamentos Irregulares, Rede Hidrica e Áreas de risco Geológico em uma porção da Zona Oeste de São Paulo

Figura 1

Distribución de las favelas y las parcelaciones irregulares en el Municipio de São Paulo. En detalle, la superposición de riesgo y red hídrica en una zona al oeste de la ciudad Fuente: Centro de Estudos da Metrópole (2016) y Geosampa (2017)

Elaboración propia

En medio de este contexto, de formación y multiplicación de la precariedad urbana, a partir del 2000 el Estado brasileño comenzó a implementar y financiar un conjunto de políticas sociales y urbanas para enfrentar este cuadro. Los programas de urbanización de favelas ganaron entonces espacio en las agendas municipales y, de forma original, comenzaron a implementar acciones en las que intercalaron las dimensiones urbanística, habitacional, social y jurídica; asimismo, articularon los diversos entes federativos para promover e implementar sus acciones, pues es en el plano local en el que estos programas se ejecutan.

Este texto ha tomado como punto de partida las acciones públicas de urbanización de favelas realizadas a partir del 2000 en dos favelas del Municipio 
de São Paulo:Vila Nova Jaguaré y Sapé. Se ha procurado responder hasta qué punto las obras de urbanización mantuvieron las directrices de las políticas y programas públicos, a la luz de dos cuestiones centrales: ¿la favela se volvió ciudad? ¿Y cuáles son los nuevos rostros de la precariedad ${ }^{5}$ ?

\section{EL PROBLEMA FAVELA EN EL BRASIL}

Para comprender mejor las recientes acciones públicas de intervención en favelas, de São Paulo, presentamos brevemente el historial de los programas de urbanización de favelas implementados por el Estado brasileño, pues fue a partir del conocimiento acumulado y de las acciones públicas a lo largo de los años que se construyeron los programas actuales.

Los programas y las acciones públicas de intervención en favelas pueden describirse y dividirse en tres periodos, en consideración al contexto sociopolítico y económico de cada uno de ellos: 1) hasta el final de la década de 1960, con las políticas de remoción de favelas; 2) desde mediados de la década de 1970 hasta el final de 1990, con acciones puntuales de dotación de infraestructura y de apoyo para la mejoría de la vivienda; 3) a partir del 2000, cuando los programas se institucionalizan como política pública y ganan escala y cobertura en el territorio nacional.

Hacia finales de 1960, la respuesta dada para el problema favela fue principalmente la remoción. Hasta entonces, la favela se tenía como un problema urbano que debía ser erradicado de las ciudades. En las primeras décadas del siglo xx hubo remociones con el pretexto de abrir espacio para los proyectos de mejoramiento de las ciudades; más tarde, en los primeros años del Gobierno militar, se dieron remociones de favelas localizadas en barrios centrales (tanto totales como parciales), acompañadas de la reubicación de

5 Este texto es fruto de la investigación Intervenciones urbanas contemporáneas en ciudades de América Latina, realizada en el Centro de Apoyo a la Investigación, Producción y Lenguaje del Ambiente Construido (Napplac) de la Facultad de Arquitectura y Urbanismo de la Universidad de São Paulo (FAUUSP), que por más de tres años siguió y registró el proceso de urbanización y la posurbanización de estas dos favelas. 
las poblaciones de estas favelas a grandes conjuntos habitacionales, por lo general, en periferias distantes de las metrópolis ${ }^{6}$.

Entre mediados de la década de 1970 y finales de la década de 1990 se constituyeron programas que buscaban solucionar problemas puntuales. Como ejemplo de esto, aún bajo el Gobierno militar (entre 1975 y 1985), los programas de dotación de infraestructura (agua y energía eléctrica), de financiamiento de lotes urbanizados y de construcción (embrión) tenían como objetivo la ampliación o la mejoría de la vivienda, en paralelo con la producción y comercialización de la habitación popular, aunque siempre por debajo de la demanda popular. A mediados de 1980, con la apertura democrática, se amplió la reivindicación por la regularización de favelas y por la provisión de viviendas populares. Algunos Gobiernos progresistas implementaron programas de urbanización, en contra de las políticas de liberalización económica que se intensificaron en esa época.

En 1990 los programas se ampliaron. Amparados en los principios de la nueva Constitución Federal' ${ }^{7}$, los programas de urbanización de favelas (slum upgrading) ganaron una dimensión mayor y se multiplicaron debido a los financiamientos multilaterales, como los proyectos de descontaminación de la cuenca de la represa de Guarapiranga (SP/Banco Mundial) y el proyecto Favela-Barrio (RJ/BID) (Cardoso, 2007).

El punto de inflexión se dio en el 2003, cuando se implementó una nueva agenda urbana que reforzó el papel del Estado como formulador y financiador de políticas sociales. Los programas de urbanización de favelas entraron con fuerza en las agendas públicas, amparados en la nueva organización políticoinstitucional dada por la creación del Ministerio de las Ciudades (2003), los

6 El Gobierno militar (1964-1985) implementó la política nacional de habitación y, conjuntamente, el Sistema Nacional de Habitación (SNH), además del Banco Nacional de Habitación (BNH). Entre 1964 y 1973 esta política se orientó hacia la remoción de favelas y la construcción de habitación popular en las periferias de las metrópolis.

7 La Constitución Federal del Brasil (1988) definió los principios de la función social de la ciudad y de la propiedad en el capítulo específico de política urbana (artículos 182 y 183), que 13 años después fueron reglamentados por el Estatuto de la Ciudad (Ley 10.257, 2001). 
Programas de Aceleración del Crecimiento ${ }^{8}$ y el Programa Mi Casa MiVida ${ }^{9}$. En ese momento, las acciones públicas buscaron integrar las dimensiones urbanística, habitacional, jurídica y social con la intención de alcanzar la regularización urbanística y rural.

Con la creación y la implementación de programas y financiamientos federales para la urbanización de favelas, se logró un gran avance en el enfrentamiento de la precariedad urbana y habitacional. Las experiencias realizadas, además de avanzar en los aspectos institucionales y de reestructurar las políticas urbanas y habitacionales, articularon los diversos entes federativos para promover e implementar acciones públicas en las favelas, al mismo tiempo que se progresó en la capacitación de los municipios.

Si las acciones públicas de intervención en favelas se iniciaron con políticas de remoción, durante las décadas de 1980 y 1990 fueron experimentando con planes innovadores. El 2000, a su vez, marcó el inicio de acciones complejas y programas de gran alcance en el territorio nacional. Pero, aunque se haya avanzado mucho, la favela aún continúa siendo un problema - sea por su extensión en el territorio nacional o por la dimensión poblacional que alberga - . De forma recurrente queda como solución la remoción parcial o integral, especialmente en áreas de interés inmobiliario, obras públicas viales o de canalizaciones de cursos de agua, y principalmente en obras de urbanización de favelas y lotizaciones irregulares (Zuquim, 2012).

\section{¿Y EN SÃO PAULO?}

Si el Brasil experimentó grandes avances jurídicos, institucionales y programáticos en el campo de la política urbana y habitacional, São Paulo también

8 El Programa de Aceleración del Crecimiento (PAC) fue creado en el 2007 para estimular el crecimiento de la economía por medio de obras de infraestructura social, urbana, logística y energética. El Programa de Urbanización de Asentamientos Precarios (PAC UAP) es un componente de los ejes de infraestructura social y urbana.

9 El Programa Mi Casa Mi Vida fue creado en el 2008 para mitigar los efectos internos de la crisis económica internacional —crear empleo y estimular la industria de la construcción civil— a través de la construcción de viviendas para familias con ingresos de hasta 10 salarios mínimos. 
se mostró pionero en la construcción de políticas y acciones de urbanización de favelas. Sin embargo, una de las características resaltantes de las ciudades brasileñas es la discontinuidad de las políticas sociales, entre ellas las urbanas y habitacionales, pues estas dependen de la estructura político-institucional de cada municipio y, al mismo tiempo, de una buena articulación política entre los diversos entes federativos ${ }^{10}$.

Las acciones públicas de urbanización de favelas a veces avanzan y garantizan sus derechos con un Gobierno progresista, y a veces retroceden con un Gobierno conservador. En São Paulo, la alternancia de gestiones municipales de líneas programáticas distintas fue recurrente y los programas de urbanización de favelas se movieron entre avances y retrocesos.

A inicios de la década de 1980, se empezaron a implementar en la ciudad las acciones públicas de urbanización de favelas, en oposición a la directriz remocionista, que era la regla hasta entonces. Pero estas no duraron mucho tiempo, los programas fueron interrumpidos y se retomó la política de la remoción, especialmente en áreas pudientes. En la década siguiente se dieron nuevas idas y venidas. A inicios de 1990, con un Gobierno progresista, se rescataron los programas de urbanización de favelas, pero a mediados de la misma década se invirtió el sentido y las políticas de remoción retomaron su protagonismo.

Con la llegada del 2000, hubo una inflexión en la acción pública de urbanización de favelas en un momento de consolidación de los avances de las políticas sociales - especialmente urbana y habitacional-, así como de reanudación y articulación de los espacios de participación social. Las acciones de este periodo se dividieron en dos momentos: el primero, entre el 2001 y el 2004, con la elaboración de programas y proyectos; y el segundo, a partir del 2006, con la implementación de las obras.

En un primer momento, a partir del 2001, regresaron los programas de urbanización de favelas y provisión habitacional. Se creó el Programa Barrio

10 La República Federativa del Brasil está formada por la unión indisoluble de los estados y municipios, y el Distrito Federal (art. 1 de la CF 1988). 
Legal ${ }^{11}$, cuya meta era la recategorización urbana y ambiental de áreas en precariedad. Tal programa recuperó las experiencias exitosas de 1990 en São Paulo para ofrecer acciones públicas y avanzar en ellas. El programa buscó integrar los diversos proyectos habitacionales en ejecución y, de forma inédita, interconectar e implementar una política de riesgo urbano ambiental vinculada a los programas de urbanización y demás políticas sectoriales. De esta manera, sería posible una actuación a gran escala a partir de la licitación de proyectos y obras, sin perder con esto el espacio para la participación popular (Samora; Zuquim, 2017).

En un segundo momento, a partir del 2006, se dio continuidad a los proyectos de urbanización, ya que estos estaban licitados. En paralelo, se reorganizó la política de habitación social del municipio con la creación del Plan Municipal de Habitación (PMH) para 2009-2024 y del Programa de Urbanización de Favelas (São Paulo, 2010), esta vez como respuesta a las exigencias federales para la captación de recursos del PAC y del Programa Mi Casa Mi Vida. Paradójicamente, al mismo tiempo en que las obras de urbanización avanzaban en escala y cobertura territorial, se retomaron las acciones de desalojo en algunas favelas de la ciudad, principalmente donde estaban previstas obras viales.

De esta forma, el PMH del 2009, que tenía una meta ambiciosa de atención de 800000 familias en la capital, alcanzó algo más del $18 \%$ de la cifra que deseaba en sus primeros cuatro años (incluyendo al resto de los programas habitacionales), y en los años siguientes avanzó a pasos aún más lentos (Zuquim, 2012). Como ejemplo de ello, el resultado presentado por el programa hasta el 2012 fue la atención de algo más de 82000 familias a partir de obras concluidas o en ejecución (Prefeitura Municipal de São Paulo, 2012). Ya entre el 2013 y

11 El Programa Barrio Legal proponía la recategorización de áreas territorialmente definidas en regiones donde predominaba la precariedad habitacional y urbana. Incluía la urbanización y regularización rural de favelas y lotizaciones; reforma y regularización de conjuntos habitacionales; provisión habitacional e implantación de equipamientos públicos y comunitarios. Pretendía también integrar las diversas acciones habitacionales en curso en la ciudad y, al mismo tiempo, permitir una actuación a gran escala a partir de la licitación de proyectos y obras, sin perder con esto el espacio para la participación popular (Samora, 2010). 
el 2016 se concluyeron obras que beneficiaron a 14114 familias, incluyendo aquellas iniciadas en el periodo anterior (Habitasampa, 2016b).

Se debe destacar que la crisis económica e institucional instaurada en el Brasil a partir del 2014 comprometió la transferencia de recursos federales; y los programas habitacionales tuvieron una reducción en su ritmo de ejecución, así como en la provisión habitacional.

Aunque se lanzó en junio del 2016 un nuevo Plan Municipal de Habitación, este aún no se ha implementado, por tanto, el municipio aún sigue el Programa de Urbanización de Favelas del Plan Municipal de Habitación para 2009/2024.

Como ya se mencionó, la discontinuidad del poder local y en consecuencia de los programas de urbanización de asentamientos precarios ocasiona avances y retrocesos. Aunque se hayan realizado varias experiencias de intervención a gran escala y con amplia cobertura territorial, continúan marcadas por un discurso programático dispar sobre las acciones públicas efectivas de intervención en asentamientos precarios (Zuquim, 2012).

\section{EL Programa de URBANIZACIÓN DE FaVELAS EN SÃO PAULO: LAS EXPERIENCIAS DE LAS FAVELAS VILA NOVA JAGUARÉ Y SAPÉ}

Si la alternancia en el poder impactó directamente en las directrices de las políticas públicas y acciones programáticas, esto no sería diferente con los proyectos y obras derivados de ellas. Entonces, para evaluar si las favelas se integraron a la ciudad y si surgió una nueva precariedad urbana, se tiene que relacionar el programa de urbanización con las acciones derivadas para situar las intervenciones en su contexto.

De esta forma, se observa con más atención el Programa de Urbanización de Favelas. Surgido como una continuación del Programa Barrio Legal, para la ejecución de las obras de proyectos licitados, y como respuesta a las exigencias federales, se diseñó con el objetivo de solucionar las cuestiones de riesgo ambiental, así como para proveer infraestructura urbana y unidades habitacionales. 
El diseño del Programa de Urbanización de Favelas se estructuró por medio de tres componentes fundamentales: las acciones de reurbanización, la intervención infraestructural y la intervención de carácter ambiental.

Las intervenciones de reurbanización se dieron en la remoción total y la provisión de las unidades habitacionales, motivadas por el interés público, incendio o riesgo urbano ambiental en las favelas.

En el componente infraestructural, las acciones se destinaron a la dotación de infraestructura, servicios y equipamientos urbanos; la remoción por riesgo urbano ambiental; la priorización del mantenimiento de las características de la parcelación del suelo y de las unidades habitacionales; y la provisión de unidades nuevas (interna y externamente al perímetro de la intervención).

Las intervenciones de carácter ambiental se dieron en obras de rectificación del cauce de riachuelos, la implementación de parques lineales o áreas de esparcimiento, dotación de infraestructura, mantenimiento parcial de las características de la parcelación del suelo y de las unidades habitacionales, remociones derivadas del riesgo urbano ambiental, y provisión de unidades nuevas.

Como objeto del análisis, se presentan los proyectos implantados en la favela Vila Nova Jaguaré (infraestructural) y en la favela del Sapé (ambiental), que son emblemáticos en el Programa de Urbanización de Favelas y presentan transformaciones físico-urbanísticas relevantes.

La favela Vila Nova Jaguaré fue objeto de varias obras de intervención pública a lo largo de su historia (su ocupación data de la década de 1960). La intervención más reciente comenzó en el 2001, aún en el ámbito del Programa Barrio Legal. Su diseño priorizó la estabilización de laderas y tomó como plan urbano la creación de grandes áreas libres, así como la dotación de infraestructura vial, sanitaria y eléctrica.

La obra empezó en el 2006, dentro del Programa de Urbanización de Favelas. $\mathrm{Y}$, a pesar de presentar alteraciones en relación con el proyecto original, las obras continuaron las siguientes directrices del programa: dotación de infraestructura y universalización de los servicios urbanos, eliminación de las áreas de riesgo, integración de la favela a los barrios vecinos y regularización rural para garantizar la permanencia en el lugar. 
La figura 2 presenta el estado de la favela en dos momentos: situación inicial (2001) y con el proyecto de intervención implementado (2006).

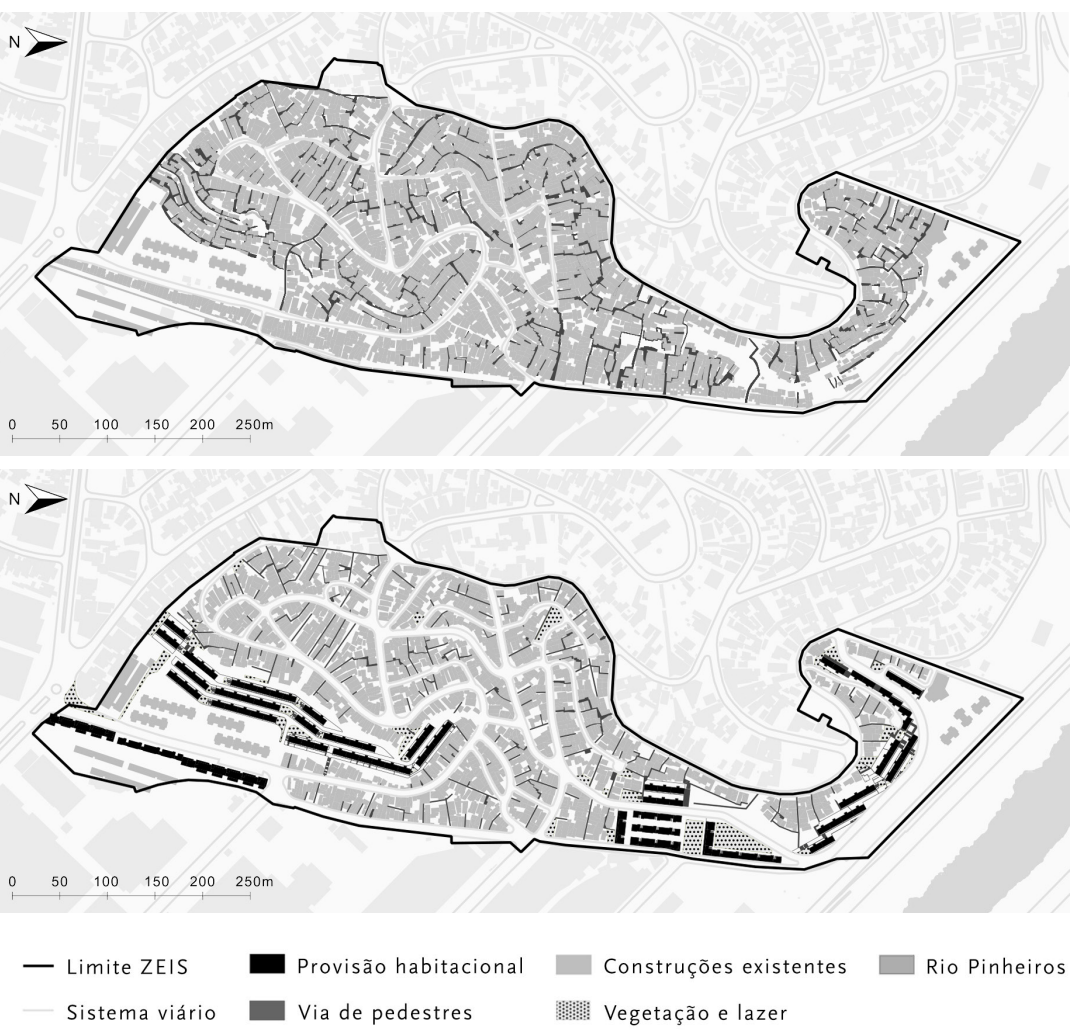

Figura 2

La favela Vila Nova Jaguaré: situación inicial y con proyecto de intervención Fuente: Zuquim, Mazo y Mautner (colaboradora) (2017) 
En términos de provisión habitacional, se entregaron 942 unidades internas y externas al perímetro de la intervención, y se removieron a 1879 familias (52\% de los domicilios registrados) para la implantación de infraestructura y eliminación de riesgo geotécnico; por tanto, 937 familias, de una población total de 3600 familias, se quedaron sin atención habitacional en nuevas viviendas. Una primera observación sobre el emprendimiento muestra que el saldo entre remoción y provisión permanece como un pasivo no solucionado.

La implantación de infraestructura y la reorganización del sistema vial facilitaron y diversificaron las actividades comerciales por la llegada de correos, carnicerías, bancos, casas de loterías, cabinas de internet, dentistas, mercadillos, etcétera.

La contención geotécnica tuvo como resultado extensas áreas libres. Estas, en gran parte, fueron "organizadamente" reocupadas, ya sea por el crimen una actividad rentable en esas circunstancias o por la población afectada por las obras, de modo que se inició un nuevo ciclo de irregularidades urbanas y jurídicas (figura 3). Se debe destacar que el saldo residual entre remoción y previsión abrió el camino para nuevas ocupaciones y las antiguas áreas, originalmente clasificadas de riesgo, fueron reocupadas.

En la favela del Sapé se observa el protagonismo del componente ambiental, pues su ocupación tuvo como elemento estructurante el riachuelo del Sapé. Las primeras ocupaciones datan de la década de 1970 y se localizaron en las áreas remanentes de lotizaciones cercanas y sobre el riachuelo, en palafitos.

El proyecto de la intervención se inició en el 2010 y permanece en ejecución. En esa ocasión, aproximadamente 7600 personas habitaban el área en poco más de 2500 inmuebles (información obtenida en una visita técnica al local en el 2016). Como plan urbano, se preveía la canalización del riachuelo con el uso de gaviones y la delimitación de un corredor non æadificandi de 1,5 veces el ancho del canal (Troncoso, 2010) ${ }^{12}$.

12 Fuera del perímetro de la intervención se realizaron también obras de canalización del riachuelo y la creación de un parque lineal en el ámbito de los programas Riachuelo Limpio y 100 Parques, de los Gobiernos estadual y municipal. Ver más en Travassos (2010). 

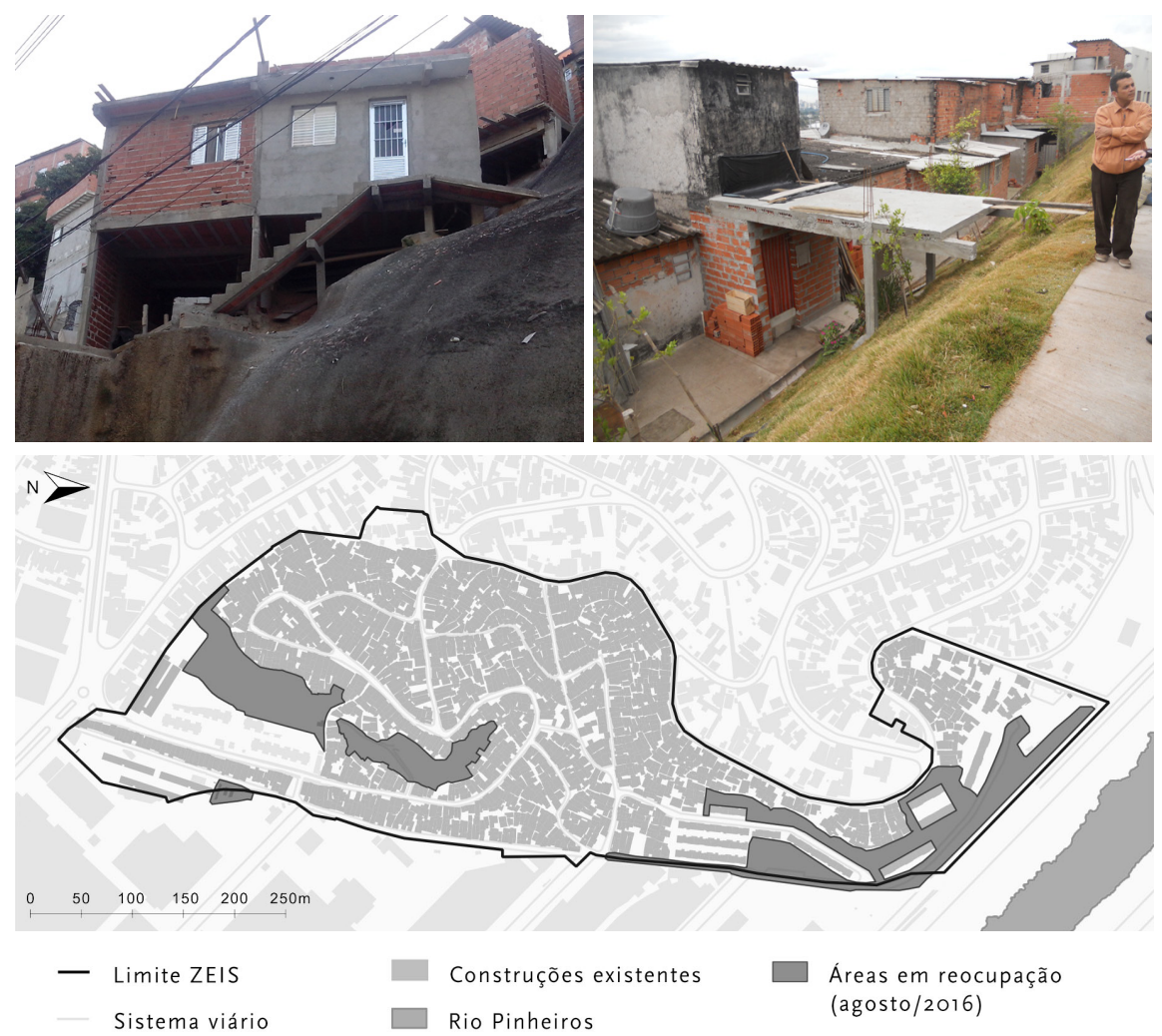

Figura 3

Construcciones ex-post ocupación de las áreas libres, paseos y contenciones, y mapa de las reocupaciones

Fuente: Zuquim, Mazo, Mautner (colaboradora) (2017)

Fotografías: Maria de Lourdes Zuquim (2015)

Además, se proyectó la instalación de infraestructura sanitaria y eléctrica en las áreas remanentes, el rediseño de partes del sistema vial, la implantación de áreas libres para esparcimiento y la construcción de nuevas unidades habitacionales. Para esto, se planeó la remoción de 1117 familias (45\% del total registrado). La provisión habitacional ya concluida era de 462 unidades habitacionales dentro 
del perímetro de la intervención; por tanto, casi 650 familias se quedaron sin atención habitacional en nuevas viviendas. De estas, una parte recibió indemnizaciones; y la otra, un subsidio para alquiler mientras se mantienen a la espera de próximas unidades, sin fecha de entrega aún.

En la figura 4 se observan también dos momentos de la favela del Sapé: el registro (2010) y el proyecto de intervención (2012). Se nota que en el proyecto ejecutado el patrón morfológico se alteró por una nueva vía transversal al riachuelo y la apertura de vacíos como consecuencia de la verticalización, aunque estos vacíos se transformaron en propiedad privada de los condominios al ser enrejados o cercados. A pesar de estar incluidas en el proyecto, la obra de urbanización redujo las áreas verdes y de esparcimiento, debido a problemas rurales y técnicos. Las áreas remanentes, donde se localizan las construcciones existentes (figura 4), tienen un diseño urbano alterado solo lo suficiente como para recibir infraestructura urbana. Es decir, la implantación de la obra deja transformaciones urbanas evidentes, como la intervención sobre el riachuelo (figura 5), pero también cuestiones aún no resueltas, como el saldo entre remoción y provisión.

Finalmente, la cuestión ambiental es tratada de forma parcial en la intervención. El diseño del programa no alcanzó la escala de intervención en la cuenca o microcuenca como unidad de planeamiento. Los desequilibrios hídricos, tales como desbordes e inundaciones, no fueron tratados efectivamente en la intervención. 


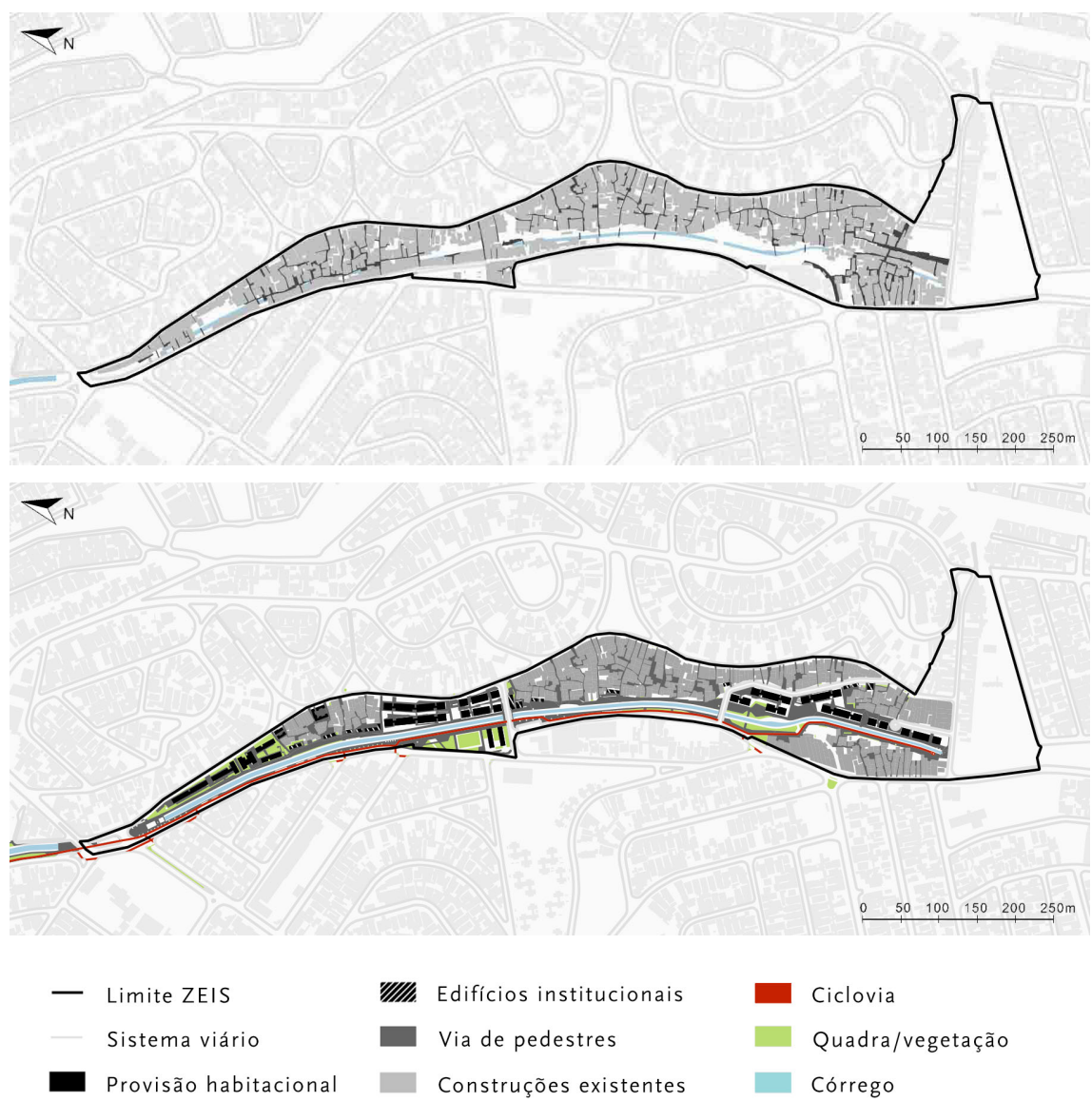

Figura 4

La favela del Sapé: situación inicial y proyecto de intervención Fuente: Zuquim, Mazo, Mautner (colaboradora) (2017) 

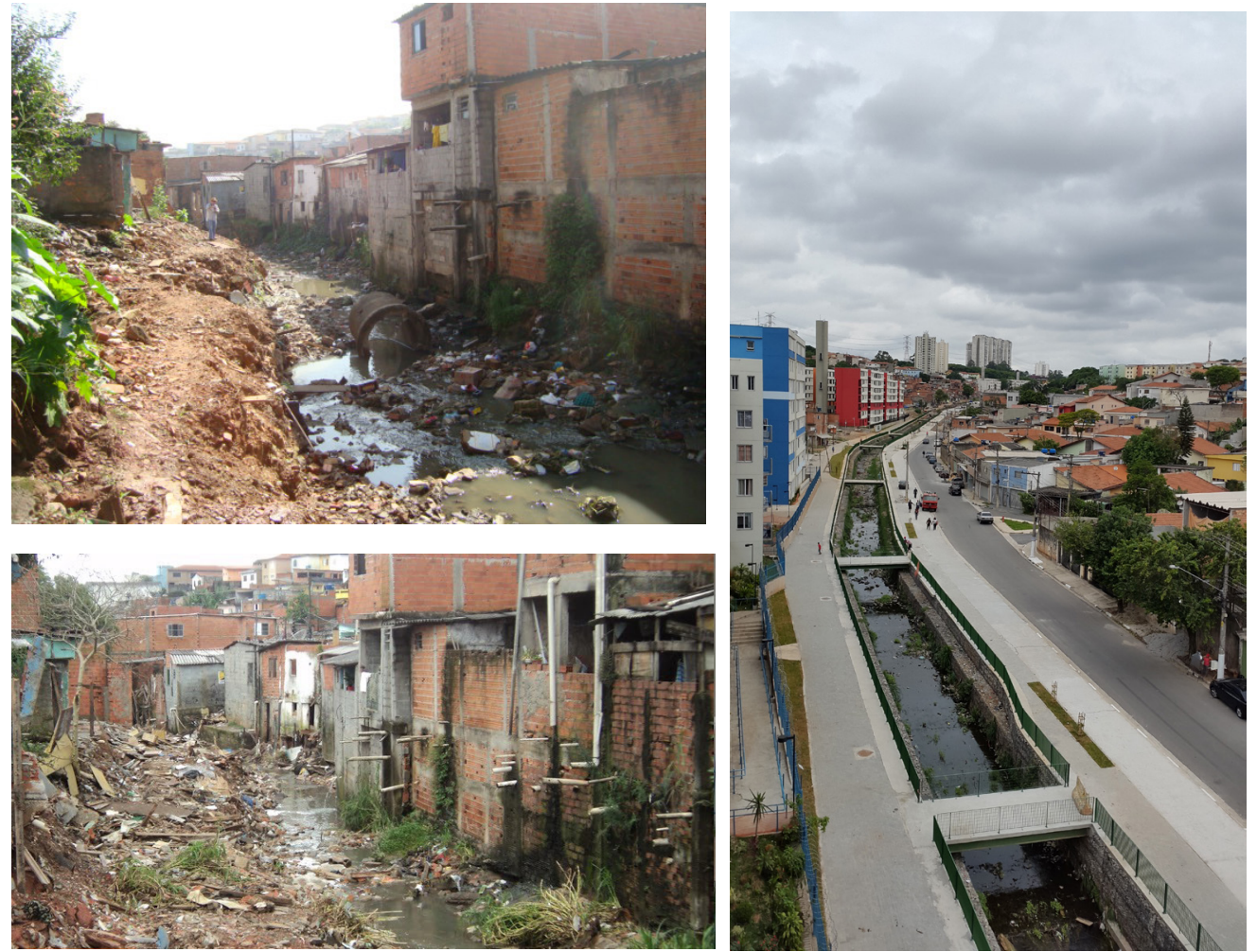

Figura 5

Riachuelo del Sapé antes y después de la intervención

Fuente: blog Micro Rede Sapé (2012); Ana Júlia Brandão (2015)

\section{SÃO PAULO: ¿VIEJAS NUEVAS FAVELAS?}

La reciente transformación urbana en las favelas Vila Nova Jaguaré y Sapé muestra que las directrices del Programa de Urbanización de Favelas aún no han sido homologadas con las acciones efectuadas en la urbanización, especialmente las de permanencia de las familias en el lugar, universalización de la infraestructura y los servicios urbanos, y eliminación de las áreas de riesgo. 
En primera instancia, aunque la articulación entre remoción de viviendas y provisión habitacional figure al menos en la teoría como premisa en los programas de urbanización, en los casos de Vila Nova Jaguaré y del Sapé el programa no logró el éxito en este asunto, y el saldo residual en la demanda por vivienda fue significativo. Las remociones fueron notoriamente mayores que las provisiones habitacionales internas y externas. Son más de 900 familias en el Jaguaré y casi 650 familias en el Sapé sin atención, y en ambos casos estas familias se han quedado durante años en la modalidad de subsidio para alquiler a la espera de sus viviendas.

Las intervenciones crearon nuevas localizaciones urbanas, y una consecuente valorización inmobiliaria - aumento notorio del precio de las viviendas tanto para la venta como para el alquiler- calentó el mercado interno de la favela y valorizó el entorno. Por ello, las casas se verticalizaron y se subdividieron para albergar más unidades habitacionales o actividades de comercio.

Las nuevas áreas libres e institucionales, creadas por la urbanización, han sido organizadamente reocupadas por viviendas. Estas áreas, que en su mayoría eran antiguas zonas de riesgo, vuelven a ser habitadas como memoria atávica del lugar que recurrentemente vuelve a su origen: la vivienda. Es importante destacar que estas áreas fueron proyectadas y articuladas espacialmente con obras de consolidación geotécnica, pero el proceso de reocupación está creando nuevas áreas de riesgo geotécnico en la favela.

Esta nueva dinámica urbana tiene conexiones con nuevos agentes que han entrado en la disputa. El crimen, que siempre ocupó los espacios donde el Estado no está presente, gana nuevos contornos en la expresión de su poder y captura para sí mismo los beneficios de la mejoría urbana realizada por el Estado.

La implantación de infraestructura en las áreas remanentes de la favela trajo nuevas obligaciones financieras para el pago de los servicios urbanos: agua, luz e impuestos. Estas nuevas obligaciones financieras llegaron también para quienes se establecieron en las nuevas unidades habitacionales, que además se sumaron al financiamiento de la unidad. Las familias que no consiguen cumplir con estos pagos terminan comercializando sus viviendas, aunque de forma irregular. La diferencia entre el programa y la acción pública es nítida 
aquí; la universalización de los servicios urbanos depende de políticas sociales previstas, en teoría, en el programa de urbanización, aunque las concesionarias de los servicios públicos no aplican las debidas tarifas sociales.

Las acciones públicas insertaron la favela en el mercado urbano, pero sin vincular a sus habitantes con la protección social; es decir, fue una inclusión relativa de los derechos sociales, que recrea la precariedad urbana.

Finalmente, la escasa provisión de vivienda, la valorización inmobiliaria, el proceso de reocupación de las áreas libres y los costos de la formalización de los servicios urbanos están creando, al mismo tiempo, una nueva precariedad urbana en la favela urbanizada y la movilidad entre favelas (los que no consiguen pagar los costos de la urbanización buscan favelas más distantes, aún no urbanizadas).

Aun habiendo el país experimentado un momento de euforia, dada por la nueva política urbana y habitacional, las experiencias recientes continúan estando marcadas por el viejo patrón de urbanización desigual. La garantía del derecho a la vivienda digna, a la justicia social y a la sostenibilidad ambiental inscrita en el Programa de Urbanización de Favelas no se refleja en la acción pública reciente, lo cual marca un claro desfase entre los objetivos declarados y las acciones efectuadas en las intervenciones de urbanización de favelas.

\section{REFERENCIAS}

Bonduki, N. (1998). Origens da habitação social no Brasil. São Paulo: Estação Liberdade.

Cardoso, A. (mayo del 2007). Urbanização de favelas no Brasil: revendo a experiência e pensando os desafios. XVII Enanpur, Belén. Anais do XII Encontro Nacional da ANPUR. Belém: ANPUR, v. 1.

Cardoso,A. (2016). Assentamentos precários no Brasil: discutindo conceitos. In M. Morais, C. Krause, y V. C. Lima Neto (Eds.), Caracterização e tipologia de assentamentos precários: estudos de casos brasileiros (pp. 29-52). Brasilia: IPEA. 
Centro de Estudos da Metrópole. (2016). Relatório 2: Estimativas relativas à precariedade habitacional e ao Déficit Habitacional no Município de São Paulo - SEHAB/PMSP e CEM/CEPID. Recuperado de http:// www.fflch.usp.br/centrodametropole/upload/arquivos/relatorio2. CEMSehab2016.pdf

HabitaSampa. (2016). Atendimentos realizados. Recuperado de http:// www.habitasampa.inf.br/atendimento/atendimentos-realizados/

Instituto Brasileiro de Geografia e Estatística. (2010). Censo 2010. Brasilia: IBGE. Recuperado de http://www.ibge.gov.br/home/presidencia/ noticias/imprensa/ppts/00000015164811202013480105748802.pdf.

Mautner,Y. (1990). The Periphery As a Frontier for the Expansion of Capital (tesis de doctorado). University College de Londres, Reino Unido.

Micro Rede Sapé (sexta-feira, 2 de março de 2012). O Sapé de Ontem! Blog. Recuperado de http://microredesape.blogspot.com/2012/03/osape-de-ontem.html? $\mathrm{m}=0$

Ministerio de las Ciudades de Brasil. (2010). A urbanização de favelas: a experiência do PAC. Brasilia: Secretaria Nacional de Habitação.

Pasternak, S. (1997). Favelas e cortiços no Brasil: 20 anos de pesquisas e políticas. Cadernos de Pesquisa do LAP. São Paulo, (18).

Pasternak, S. (2006). São Paulo e suas favelas. PÓS-, (29), 176-197. doi:10.11606/issn.2317-2762.v0i19p176-197.

Prefectura de São Paulo. (2017). Mapa digital da cidade de São Paulo. Geosampa. Recuperado de http://geosampa.prefeitura.sp.gov.br/ PaginasPublicas/_SBC.aspx

Samora, P.-R., y Zuquim, M.-L. (2017). Da luta pela moradia à urbanização de assentamentos precários: a politica habitacional no Brasil. En M. L. Zuquim y L. M. Sánchez Mazo (orgs.), Barrios populares Medellín: favelas São Paulo. São Paulo: FAUUSP.

Samora, P.-R. (2010). Projeto de habitação em favelas: especificidades e parâmetros de qualidade (tesis doctoral). Universidad de São Paulo, Brasil. 
Secretaria da Habitación e Desenvolvimento Urbano de São Paulo. (2010). Plano municipal de habitação 2009-2024. São Paulo: Autor.

Secretaría da Habitación y Desarrollo Urbano de São Paulo. (2012). Plano municipal de habitação: a experiência de São Paulo (vol. 1). São Paulo: Superintendencia de Habitación Popular.

Travassos, L. (2010). Revelando rios: Novos paradigmas de intervenção em fundos de vale urbanos na cidade de São Paulo (tesis doctoral). Universidad de São Paulo, Brasil.

Troncoso, Ú. (2010). Reurbanização da favela do Sapé com recuperação de córrego, em São Paulo, por Base 3 arquitetos. Recuperado de http:// www.au.pini.com.br/arquitetura-urbanismo/234/reurbanizacao-dafavela-do-sape-base-3-arquitetos-sao-296129-1.aspx

Zuquim, M.-L. (2012). Urbanização de assentamentos precários no município de São Paulo: quem ganha e quem perde? En II Enanparq. Natal Anais do II Encontro Nacional da ANPARQ. NATAL: ENANPARQ, 2012. v. 1.

Zuquim, M.-L., Mazo, L.-M., y Mautner, Y. (colaboradora)(2017). Barrios populares Medellín: favelas São Paulo. São Paulo: FAUUSP. 
